\title{
DERECHOS HUMANOS, DISCAPACIDAD E INCLUSIÓN SOCIAL
}

\author{
HUMAN RIGHTS, DISABILITY AND SOCIAL INCLUSION
}

Guillermo César Vega Espejo*

\section{Resumen:}

Este documento presenta un análisis de la discapacidad y la inclusión social como un tema de derechos humanos; describimos los tres modelos que se han establecido para entender la evolución histórica en la manera de abordar el tema y cómo en el camino se han ido venciendo una serie de paradigmas y prejuicios que contrastaban con la realidad social de las personas con discapacidad en cuanto a su valor como personas con potencialidades y talentos que no eran aprovechados o eran invisibles. Se hace una reflexión de la situación contemporánea de las personas con discapacidad, tocando especialmente a la niñez y las dificultades que aún hoy encuentran así como se expone el gran reto de los Estados para diseñar políticas, programas y proyectos capaces de derrotar los maltratos aún vigentes y para que se construyan sistemas en los que prime la igualdad de oportunidades.

Palabras clave: Derechos humanos, discapacidad, inclusión social.

\begin{abstract}
:
This document presents an analysis of disability and social inclusion as a human rights issue; we describe the three models that have been established to understand historical evolution in how to address the issue and how along the way a series has been defeated of paradigms and prejudices that contrasted with the social reality of people with disabilities in terms of their value as people with potentialities and talents who were not harnessed or were invisible. A reflection is made of the contemporary situation of people with disabilities, especially touching children and the difficulties they still encounter today, as well as the great challenge of the States to design policies, programs and projects capable of defeating the mistreatment still in force and for systems to be built in which equal opportunities prevail.
\end{abstract}

Key words: Human rights, disability, social inclusion.

Presidente del Instituto Pro Desarrollo de la Persona con Discapacidad INPRODESDI. Ex Presidente del Concejo para la Integración de la Persona con Discapacidad - CONADIS. 


\section{I.TRES MODELOS A LO LARGO DE LA HISTORIA}

Lo primero que debemos tener claro es que cuando hablamos de la inclusión social de las personas con discapacidad y el efectivo respeto de sus derechos, estamos hablando, por excelencia, de un tema de derechos humanos.

Desde el origen del mundo, el abordaje sobre la situación de las personas con discapacidad ha variado considerablemente, pasando por tres modelos claramente diferenciados: un modelo de "prescindencia", otro de carácter "rehabilitador" y el actual modelo de "carácter social", que encuentra sus cimientos en la Convención Internacional sobre los Derechos de las Personas con Discapacidad de las Naciones Unidas.

Nos enfocamos en el primer modelo cuando revisamos que a lo largo de la historia y hasta la década de los ochenta, la persona con discapacidad era totalmente desvalorada, se le identificaba con la mendicidad y la indigencia en algunos casos; en otros, éramos personas que no eran útiles para la sociedad, y por el contrario, representábamos una carga, éramos un estorbo para las personas que nos rodeaban y una tercera visión era que representábamos un castigo de Dios, nacíamos con discapacidad porque nuestros padres, o uno de ellos, había cometido algún pecado grave. La persona con discapacidad no salía de su casa, debía permanecer oculta, era causa de vergüenza, incluso se pensaba que éramos personas que no merecíamos vivir.

El segundo modelo tuvo una visión desde el punto de vista médico, científico. La persona con discapacidad no tenía voz ni voto sobre qué era lo mejor para él o ella, los que decidían eran los profesionales de la salud, y la solución de los problemas de nosotros los diseñaban ellos buscando una rehabilitación en el mayor grado posible. La sociedad civil de personas con discapacidad y su fuerza para proponer o sugerir qué era lo mejor para el colectivo era muy débil o nula. Este modelo se esboza luego de la primera guerra mundial al quedar un número significativo de personas con algún tipo de discapacidad.

En el plano del Derecho, Palacios y Bariffi (2007) señalan que:

... en un primer momento esto significó la implementación de políticas legislativas destinadas a garantizar servicios sociales para los veteranos de guerra con discapacidad, que reflejaba de algún modo la creencia acerca de la existencia de una obligación por parte de la sociedad, de compensar a estas personas mediante pensiones de invalidez, beneficios 
de rehabilitación y cuotas laborales. No obstante, por la década de los años sesenta dichas medidas fueron extendidas a todas las personas con discapacidad, dejándose de lado la causa de las deficiencias. (p.16)

Según Degener \& Quinn (2000) los niños con discapacidad tenían derecho a la educación, pero en escuelas separadas, las personas con discapacidad tenían derecho a la rehabilitación, pero ello incluía el control de muchas áreas de sus vidas por parte de los expertos, las medidas de acción positiva se introdujeron porque - a diferencia de otros grupos protegidosno se consideraba que las personas con discapacidad fueran capaces de trabajar por sus propios méritos.

El tercer y último modelo es el de carácter social. Este modelo va a romper esquemas pues la visión que se tenía antes de este modelo era que la integración social de la persona con discapacidad consistía en rehabilitarlo y prepararlo de la mejor manera para que pueda participar en la sociedad y así intentar hacer su vida venciendo los obstáculos existentes, luchando con las dificultades que el mundo presentaba para ellos. Se nos enseñaba a enfrentar barreras.

Este tercer modelo va a reconocer que, efectivamente, debemos estar preparados y rehabilitados de la mejor manera y que nuestra actitud debe ser la de enfrentar las dificultades que se nos presenten a lo largo de la vida pero la diferencia es que va a dar un mensaje revelador, pues si bien es cierto lo ya referido, la inclusión social de las personas con discapacidad también pasa, definitivamente, por una sociedad que nos debe tener presente en sus diseños, políticas, planes, actitudes y eliminación de barreras para que nos podamos desarrollar de la mejor manera posible; en otras palabras, una sociedad que respeta nuestros derechos humanos y que va a entender que nuestra discapacidad es mayor cuando la sociedad, el entorno, está menos preparado y adecuado para acogernos. Es entonces que van a cobrar mayor importancia los enunciados y premisas sobre la vida independiente, que se quiso impulsar en los años setenta, la no discriminación y la accesibilidad universal. Es en este tercer modelo que la sociedad civil, los gremios y asociaciones de personas con discapacidad van a ser escuchados, se va a acoger sus petitorios, como base inicial para emprender los primeros grandes cambios y a la vez las personas que la integran van a entender que tienen el deber de brindar aportes, propuestas y sugerencias para crear una sociedad inclusiva.

Según Palacios Agustina y Bariffi, Francisco José (2007), “los defensores de este modelo no son las limitaciones individuales las raíces del fenómeno, sino las limitaciones de la sociedad para prestar servicios apropiados y para 
asegurar adecuadamente que las necesidades de las personas con discapacidad sean tenidas en cuenta dentro de la organización social". (p.20)

Como ya hemos mencionado el modelo social está inmerso en la Convención de las Naciones Unidas Sobre los Derechos de la Personas con Discapacidad, instrumento que constituye un cambio de paradigma y que se compone de 50 artículos que protegen los derechos del colectivo y promueven su libertad, la igualdad y dignidad de las personas con discapacidad como sujetos de derechos, también comprende las obligaciones de los Estados Parte. Es el primer Tratado de derechos humanos del siglo XXI y se construyó con el aporte de los Estados miembros y de la sociedad civil.

Esta Convención va a impulsar que muchos países adecúen su legislación a efecto de que guarde armonía con los nuevos preceptos y enunciados a favor de las personas con discapacidad que se encuentran en este documento internacional. El Perú no ha sido ajeno a ello y por tal motivo se dio la Ley 29973, Ley General de la Persona con Discapacidad, que remplazó a la ley 27050, ley general anterior, además se han emitido sendas Resoluciones Ministeriales y Decretos Supremos con el mismo fin.

Este cambio de paradigma se resume en la consideración de la discapacidad como una cuestión de derechos humanos. A partir de dicho enfoque, las políticas ofrecidas y las respuestas brindadas a los problemas que enfrentan las personas con discapacidad pasan a ser pensadas y elaboradas desde y hacia- el respeto de los derechos humanos (Asís Roig, R. , 2004, La incursión de la discapacidad en la teoría de los derechos; Campo Cervera, 2005, Una aproximación a las nuevas líneas de fundamentación de los derechos de las personas con discapacidad; Cayo Pérez Bueno, 2004, El desmantelamiento de la discapacidad y otros escritos vacilantes, citado por Palacios, Agustina y Bariffi , Francisco, 2007 p. 11).

\section{UN TEMA DE DERECHOS HUMANOS}

Al analizar el tema que nos ocupa, no podemos dejar de señalar la importancia de la Declaración Universal de los Derechos Humanos, documento de treinta artículos que va a surgir luego de la Segunda Guerra Mundial (1939-1945), por su execrable crueldad y gran cantidad de abusos cometidos y ante la cifra de más de cincuenta millones de muertos.

La Declaración nos dice en su primer artículo que "todos los seres humanos nacen libres e iguales en dignidad y derechos y, dotados como están de razón y conciencia, deben comportarse fraternalmente los unos 
con los otros" y en el artículo segundo que "toda persona tiene todos los derechos y libertades proclamados en esa Declaración, sin distinción alguna de raza, color, sexo, idioma, religión, opinión política o de cualquier otra índole, origen nacional o social, posición económica, nacimiento o cualquier otra condición"132.

Estos preceptos, concordados con la Convención promueven a que en la actualidad la persona con discapacidad sea vista y valorada como un ser humano con igualdad de derechos y dignidad, una persona que merece que se tracen políticas transversales a su favor y que se impulse y difunda la adopción de ajustes razonables que les permitan tener una participación activa en la sociedad.

Yo me accidenté cuando tenía 16 años en 1979 y desde ese entonces, uso una silla de ruedas para desplazarme; recuerdo cuando una señora caminaba con su hijita en el sentido contrario de la acera, cuando se acercó y me observó frente a ella cruzó la pista para pasar a la vereda de enfrente; también recuerdo restaurantes elegantes a los que se me negó el ingreso en esa época.

Claro está, en aquél entonces no había un Consejo Nacional de Integración de la Persona con Discapacidad - CONADIS, no había una Comisión de Discapacidad del Congreso de la República ni un Programa de Promoción y Defensa de las Personas con Discapacidad de la Defensoría del Pueblo, tampoco existían las Oficinas Municipales de Atención a las Personas con Discapacidad - OMAPED y por cierto, no teníamos una Ley General de la Persona con Discapacidad y otras normas conexas, ni existía la Convención de las Naciones Unidas sobre los Derechos de la Persona con Discapacidad. Cierto es que hemos avanzado, tal vez no al ritmo deseado, pero hemos avanzado. La pregunta es: con todo lo logrado, ¿se respetan los derechos humanos de las personas con discapacidad en el Perú? La verdad es que la tarea es aún muy grande y no se han alcanzado los niveles esperados. Si aún hay maltrato y discriminación en Lima, ¿cuánto más será en el interior del país?

Una precisión: hasta hace poco, más de doce años, el uso del término integración era el que se utilizaba para referirse a la participación de las personas con discapacidad en diferentes campos de su vida y su participación en la sociedad. Este término se usaba en especial cuando se tocaban los temas de educación y trabajo.

132 La Declaración Universal de los Derechos Humanos es un documento que marca un hito en la historia de los derechos humanos. Elaborada por representantes de todas las regiones del mundo con diferentes antecedentes jurídicos y culturales, la Declaración fue proclamada por la Asamblea General de las Naciones Unidas en París, el 10 de diciembre de 1948 en su Resolución 217 A. 
Según García Ruiz y Fernández Moreno (2005) varios estudios concluyeron que al percibir las acciones y los programas de integración estos no cumplían las expectativas, y que era necesario un nuevo término que de alguna manera renovara el sentido de justicia y diera opciones a las personas en situación de discapacidad para participar en un mundo más equitativo, donde se reconocieran y se potencializaran sus diferencias en todos los ámbitos.

Casado y Egea (2000) señalan que en la UNESCO se planteó, de manera especial, la necesidad de buscar una definición que comprenda el real concepto de lo que era la educación inclusiva, y marcar diferencia de lo que se entendía por el término integración o de la inserción en el plan general de educación. La UNESCO ha adoptado a escala internacional el término inclusión y otros organismos internacionales lo aceptaron mostrando su utilidad. Así, este nuevo concepto de inclusión se mantuvo muy ligado con los temas y políticas relacionadas a la educación, y con el planeamiento de la enseñanza en los gobiernos a todo nivel. La inserción desde un panorama amplio y general se refería a individuos o grupos pequeños dentro del sistema actual, sin que se dedujera necesariamente que había que cambiar el sistema para posibilitar la inclusión de otros niños. Actualmente, la inclusión es entendida en todos los campos de la vida diaria y así se verifica en la doctrina de los últimos años, así como en las diferentes cartas internacionales.

Según estimaciones de UNICEF, las niñas y niños con discapacidad son uno de los grupos humanos más marginados y que sufren mayor exclusión en la sociedad. Además de enfrentar actos de discriminación en forma cotidiana, perciben actitudes negativas a la vez que las políticas públicas no se implementan y la legislación especializada no se cumple en gran porcentaje; debido a ello, el ejercicio de sus derechos en materia de salud y educación es muy limitado.

Las estimaciones sugieren que hay al menos 8 millones de niños y niñas con discapacidad menores de 14 años en América Latina y el Caribe. En comparación con los niños y niñas sin discapacidad, es frecuente que vivan en hogares pobres y es menos probable que asistan a la escuela o que sus opiniones se escuchen en la sociedad. Además, las niñas y niños con discapacidad tienen más probabilidades de crecer en instituciones residenciales, de experimentar violencia y de ser excluidos de las estrategias de prevención y respuesta a emergencias y desastres.

\section{III. ÁREAS DE TRABAJO DE UNICEF}

Con el objetivo de que cada niña y niño con discapacidad en América Latina y el Caribe pueda crecer con buen estado de salud, libre de la violencia 
y el abuso, y con acceso a la educación, a fin de que pueda desarrollar sus potencialidades, la oficina regional de UNICEF en América Latina y el Caribe se centra en las siguientes áreas:

Generación y monitoreo de datos: contribuir a la creación y el fortalecimiento de estadísticas públicas y de otros socios nacionales y regionales para obtener progresivamente más y mejores datos sobre niños y niñas con discapacidad.

Educación inclusiva: apoyar el conocimiento y la generación de evidencia sobre la educación inclusiva y contribuir a la inclusión de una perspectiva de discapacidad en los informes nacionales y regionales sobre niñas y niños que fuera de la escuela.

Desarrollo de la Primera Infancia: promocionar el diseño y la implementación de políticas y planes de desarrollo de la primera infancia integrales, intersectoriales e inclusivos.

Discapacidad y emergencia: contribuir al establecimiento, difusión e implementación de normas mínimas para abordar las necesidades de niñas, niños y adultos con discapacidad en situaciones de preparación y respuesta ante emergencias y desastres ${ }^{133}$.

Sobre la primera área de trabajo debemos señalar que no hay data disponible sobre muchos temas de niñez con discapacidad. Por ejemplo, el Poder Judicial recibe denuncias por maltratos físicos y psicológicos a niños y adolescentes ${ }^{134}$. Sin embargo, en el registro informático de denuncias no hay un link en el que se pueda consignar si el niño o la niña presuntamente agraviada es una persona con o sin discapacidad. Esto constituye un obstáculo para poder tener un ponderado, una cifra que nos permita medir el maltrato que sufre este colectivo de infantes y ello impide que se puedan elaborar políticas públicas específicas orientadas a combatir ese fenómeno. Lo mismo ocurre en los casos de niños y adolescentes víctimas de abuso sexual. Tampoco se tiene una información exacta sobre cuántos niños o adolescentes con discapacidad culminan exitosamente sus estudios de primaria o secundaria o cuántos gozan de un seguro de salud.

133 Portal web UNICEF América Latina y el Caribe. https://www.unicef.org/lac/ni\%C3\%B1os-y-ni\%C3\%B1as-condiscapacidad

134 Ley № 30364, Ley para Prevenir, Sancionar y Erradicar la Violencia Contra las Mujeres y los Integrantes del Grupo Familiar. Artículo 14. Competencia de los Juzgados de Familia.- Son competentes los juzgados de familia o los que cumplan sus funciones para conocer las denuncias por actos de violencia contra las mujeres o contra los integrantes del grupo familiar. 
Sobre la segunda área estratégica debemos señalar que en nuestro país la educación inclusiva, no llega a niveles óptimos, los centros educativos no están preparados para recibir a niños con discapacidad, hay carencia de adecuaciones arquitectónicas, faltan señales en braille para personas ciegas y señalética para personas sordas, los profesores no están preparados para recibir e instruir a niños y niñas con discapacidad, el Servicio SAANEE ${ }^{135}$ es insuficiente, no hay personal y se carece de presupuesto hasta para los pasajes. A su vez, no hay una estrategia para sensibilizar a los alumnos sin discapacidad sobre cómo deben recibir y tratar a su compañero que presente una, ello a fin que no se maltrate la dignidad de éste. Finalmente, no se trabaja en las escuelas de padres el respeto a las diferencias, la tolerancia y comprensión porque un niño con discapacidad pase a integrarse al salón en el que estudia su hijo y esto es importante pues hay muchos casos en los que los padres expresan abiertamente su malestar o incomodidad cuando un caso como el descrito se presenta, algunos han llegado a amenazar al colegio con retirar a su hijo del plantel. Se están realizando esfuerzos, pero estamos muy lejos de un resultado positivo y gratificante.

Sobre la tercera área de trabajo UNICEF, señala que las niñas y niños que no reciben la nutrición y la estimulación que necesitan enfrentan un mayor riesgo de tener un bajo nivel de desarrollo cognitivo, físico y emocional. Sostiene que, en la región de América Latina y el Caribe, 3,6 millones de niñas y niños entre 3 a 4 años no tienen un desarrollo temprano adecuado para su edad.

UNICEF encuentra una relación evidente entre los factores de riesgo para obtener un buen desarrollo en la etapa de primera infancia cuando los niños provienen de hogares pobres. Hay menos riesgo cuando se recibe una intervención y atención temprana, cuando hay un buen aprendizaje y se cuenta con los materiales adecuados para su instrucción. Los niños de escasos recursos tienen menos posibilidades de asistir a programas de educación infantil. A su vez, estas niñas y niños son más propensos a un cuidado deficiente y al castigo corporal.

Todos estos factores pueden tener un efecto capaz de perturbar el desarrollo de niños y adolescentes. A ello, tenemos que agregar que los factores de riesgo ligados a la pobreza muchas veces se dan en forma simultánea. Por lo tanto, a mayor presencia de factores de riesgo, menor

135 El SAANEE es el Servicio de Apoyo y Asesoramiento a las Necesidades Educativas Especiales. Forma parte de los Centros de Educación Básica Especial. Está conformado por personal profesional docente y no docente especializado o capacitado para brindar apoyo y asesoramiento a instituciones educativas inclusivas, a los estudiantes con Necesidades Educativas Especiales - NEE, asociadas a discapacidad, talento y superdotación, matriculados en la educación básica y educación técnico productiva, así como a los padres de familia o tutores. http://www.perueduca.pe/foro/-/message_boards/message/31303036. 
es el desarrollo de las niñas y niños. Estos factores de riesgo conducen a la desigualdad que se presentará en el desarrollo integral de los niños sobre todo en su primera infancia, lo que lamentablemente va a repercutir en su desempeño escolar y posteriormente en su vida adulta, manteniéndose así su estado de pobreza y las brechas que perjudican una adecuada inclusión social.

En el Perú no somos ajenos a ello y lo comprobamos cuando revisamos la actual situación de los Programas de Intervención Temprana - PRITE. ${ }^{136}$

Hemos alertado, desde hace varios meses, sobre un cambio discordante en las responsabilidades de los especialistas que laboran en los PRITE con la nueva norma técnica aprobada por el Ministerio de Educación, referida a la atención que deben brindar a los niños, ya que se dispone que cada profesional de las diferentes áreas de especialización se hará cargo de 12 bebés o niños, únicamente en condición de tutores, a quienes les deberán brindar todas las atenciones en conjunto, como si ese docente fuera experto en todo, atenciones que en los PRITE actualmente son brindadas por cada área, y por cada especialista, de manera individual para cada niño. Es decir, cada especialista trata al niño (psicólogo, terapista físico, terapista ocupacional, fonoaudiólogo, etc). La nueva norma técnica desnaturaliza la esencia misma del trabajo de los PRITE y exige a cada profesional, sin fundamento ni lógica alguna, que brinde todos los servicios yendo más allá de sus conocimientos. Al entenderse que esto es un disparate, muchos PRITE han establecido que cada niño reciba sólo dos terapias, las que se consideren prioritarias. Surge entonces una interrogante: ¿y si el niño necesita del concurso de más especialistas para su adecuado desarrollo? La pregunta queda sin contestar.

El espíritu del PRITE es el trabajo coordinado e interdisciplinario que se realiza; es el que garantiza el logro del desarrollo integral e independencia y bienestar del niño de manera exitosa.

También sostenemos que la edad de los bebés y niños para que gocen del servicio y atención de los PRITE debe regresar a la edad de 0 a 5 años, como fue desde que se crearon, y no de 0 a 3 años como es en la actualidad, a raíz de la aprobación del Reglamento de la Ley 28084, Ley General de Educación,

136 Un PRITE o centro que promueve el programa de Intervención Temprana es un espacio educativo que brinda atención integral no escolarizada a niños menores de 3 años con discapacidad o en riesgo de adquirirla por antecedentes tanto biológicos como socio ambientales. La intervención temprana es importante porque permite la detección de discapacidades como el autismo, síndrome de down, parálisis cerebral, discapacidad intelectual, entre otras. Del mismo modo, contribuye a la provisión temprana de los servicios necesarios para reducir los efectos de factores que agudizan la discapacidad, así como la prevención de otros problemas. Los profesionales especializados en educación especial brindan servicios como psicología, pedagogía especial, terapia física, ocupacional y de lenguaje. 
mediante Decreto Supremo Nro. 011-2012-ED ${ }^{137}$. (Ver Art.84 párrafo b del reglamento) que reemplazó a la Directiva Nro. 081-2006-VMGP/DINEBE. Esta modificatoria no tuvo fundamento lógico alguno.

Subrayamos que es muy importante que los niños vuelvan a gozar de dos años más de esa atención tan importante, intensa, multidisciplinaria, de estrecho involucramiento a los padres, siempre y cuando el equipo multidisciplinario lo estime conveniente, a efecto de que los resultados sean más notorios, robustos, evidentes y óptimos, pedido que hacemos sin otro propósito que atender al Principio del Interés Superior del Niño, consagrado en la Convención de las Naciones Unidas Sobre los Derechos del Niño ${ }^{138}$ y en el Código de los Niños y Adolescentes. La adecuada intervención tempana es esencial.

Es ante esta situación que la Comisión de Discapacidad e Inclusión Social del Congreso de la República presentó un proyecto de ley que corregía estas deficiencias, lamentablemente al haberse disuelto el Congreso, los proyectos pasan al archivo.

Finalmente, debemos señalar que los PRITE sufren una gran carencia de materiales para poder trabajar con los niños, a lo que hay que sumar la deficiente infraestructura de la mayoría de sus locales. Este es un reto enorme, pues se trata de salvaguardar los derechos constitucionales a la salud y la educación de miles de niños peruanos.

Con relación a la cuarta área de trabajo, sobre la atención que se debe brindar en los casos de emergencia de desastres, podemos decir que en nuestro país no nos encontramos preparados. En el terremoto de agosto del 2007 se pudo comprobar que no se habían diseñado estrategias ni políticas sobre cómo brindar las primeras atenciones a las personas con discapacidad.

Diferentes poblaciones pueden sufrir riesgos similares de verse expuestas a los efectos negativos de los desastres ambientales y causados por el hombre, pero su vulnerabilidad real depende de sus condiciones socioeconómicas, su empoderamiento cívico y social y su acceso a recursos de mitigación y socorro. Las personas con discapacidad se ven afectadas de manera desproporcionada en situaciones de desastre, emergencia y conflicto debido a que las medidas de evacuación, respuesta (incluidos los refugios,

\footnotetext{
137 Art.84.- b) Programa de Intervención Temprana PRITE Brinda atención no escolarizada a los niños menores de tres años con discapacidad o en riesgo de adquirirla...///

138 Artículo 3 1. En todas las medidas concernientes a los niños que tomen las instituciones públicas o privadas de bienestar social, los tribunales, las autoridades administrativas o los órganos legislativos, una consideración primordial a que se atenderá será el interés superior del niño.
} 
los campamentos y la distribución de alimentos) y recuperación les resultan inaccesibles ${ }^{139}$.

Como sabemos, Perú se encuentra en una fuerte zona sísmica, a ello hay que agregar los huaicos que se presentan durante el verano y las fuertes lluvias que tenemos en la sierra y selva del país, y que están causando cada vez más inundaciones en centros poblados y comunidades. Si bien es cierto que el Instituto de Defensa Civil - INDECI, publicó en el año 2016, un manual denominado Derechos de la Persona con Discapacidad en Casos de Emergencia de Desastres, lo cierto es que un considerable grupo de la sociedad civil considera que resulta incompleto y necesita correcciones. Se requiere la elaboración de protocolos que sean de dominio y conocimiento de los operadores de la salud, bomberos, policía, serenazgo y brigadistas de las instituciones públicas y privadas.

En el artículo 11 de la Convención, relativo a situaciones de riesgo y emergencias humanitarias, se presta especial atención a la obligación de los Estados Partes de adoptar "todas las medidas necesarias para garantizar la seguridad y la protección de las personas con discapacidad en situaciones de riesgo, incluidas situaciones de conflicto armado, emergencias humanitarias y desastres naturales". Además, en el artículo 4.1 se afirma que «los Estados Partes se comprometen a asegurar y promover el pleno ejercicio de todos los derechos humanos y las libertades fundamentales de las personas con discapacidad sin discriminación alguna por motivos de discapacidad», y en el artículo 32 se reconoce la importancia de la cooperación internacional con miras a hacer frente a la capacidad limitada de algunos Estados para responder a situaciones de riesgo y crisis humanitarias.

Contar con leyes y protocolos especializados en la materia es sumamente importante, se trata de brindar atención oportuna y salvar muchas vidas, lo que concuerda con el enfoque de derechos humanos que le es inherente a las personas con discapacidad.

No olvidemos que el Perú es Parte de la Convención de las Naciones Unidas Sobre los Derechos del Niño. Esta Convención fue proclamada y adoptada por la Asamblea General de la ONU el 20 de noviembre de 1989. En ella se establecen los derechos inalienables de todos los niños y las niñas, pero también las obligaciones de los Estados, los poderes públicos, los padres, las madres y la sociedad en su conjunto, incluidos los propios niños y niñas, para garantizar el respeto de esos derechos y su disfrute por todos los niños sin distinción de ningún tipo.

139 NACIONES UNIDAS- Personas con Discapacidad Departamento de Asuntos Económicos y Sociales. https://www. un.org/development/desa/disabilities-es/discapacidad-desastres-naturales-y-situaciones-de-emergencia.html 
Es uno de los Tratados internacionales de derechos humanos más ampliamente respaldados, 196 naciones lo han ratificado. Estados Unidos es el único país que no ha completado el proceso de ratificación de la Convención.

Este documento consagra la protección de los derechos del niño en su artículo 23 y tal dispositivo ha inspirado a diversos Estados, a efecto de que se consignen similares enunciados en sus correspondientes legislaciones ${ }^{140}$.

Por otro lado, la Convención de las Naciones Unidas sobre los Derechos de la Persona con Discapacidad en su Artículo sétimo, señala los derechos y consideraciones que se deben tener en cuenta en el tema de la niñez con discapacidad. ${ }^{141}$

Hemos analizado tres áreas de trabajo que nos dan luces de que aún es larga la tarea por lograr el respeto a la integridad de la niñez con discapacidad y por lograr un marco de igualdad de oportunidades que asegure su adecuado desarrollo integral; es decir, aún nos falta, a cabalidad, por respetar sus derechos humanos.

\section{RECOMENDACIONES}

Diseñar y ejecutar planes y políticas públicas orientadas a lograr la inclusión social y participación activa de la niñez con discapacidad. Implementar un adecuado y eficiente sistema de monitoreo de las mismas, con indicadores y metas claras.

Aplicar una reingeniería de la educación inclusiva desde una perspectiva realista y multidisciplinaria.

Desarrollar estrategias para derrotar estigmas y prejuicios a todo nivel, no sólo con campañas de sensibilización o concienciación sino

140 Artículo 23.- 1. Los Estados Partes reconocen que el niño mental o físicamente impedido deberá disfrutar de una vida plena y decente en condiciones que aseguren su dignidad, le permitan llegar a bastarse a sí mismo y faciliten la participación activa del niño en la comunidad.

2. Los Estados Partes reconocen el derecho del niño impedido a recibir cuidados especiales y alentarán y asegurarán, con sujeción a los recursos disponibles, la prestación al niño que reúna las condiciones requeridas y a los responsables de su cuidado de la asistencia que se solicite y que sea adecuada al estado del niño y a las circunstancias de sus padres o de otras personas que cuiden de él.

141 Artículo 7: Niños y niñas con discapacidad • Los Estados Partes tomarán todas las medidas necesarias para asegurar que todos los niños y las niñas con discapacidad gocen plenamente de todos los derechos humanos y libertades fundamentales en igualdad de condiciones con los demás niños y niñas. • En todas las actividades relacionadas con los niños y las niñas con discapacidad, una consideración primordial será la protección del interés superior del niño.

- Los Estados Partes garantizarán que los niños y las niñas con discapacidad tengan derecho a expresar su opinión libremente sobre todas las cuestiones que les afecten, opinión que recibirá la debida consideración teniendo en cuenta su edad y madurez, en igualdad de condiciones con los demás niños y niñas, y a recibir asistencia apropiada con arreglo a su discapacidad y edad para poder ejercer ese derecho. 
también promoviendo las buenas prácticas y difundiendo experiencias exitosas.

Contar con un sistema de data integral e interconectado que permita obtener estadística necesaria en las diversas áreas socio-económicas y culturales de nuestro país.

Fomentar las mesas de trabajo con los padres y familiares, conscientes de que son los grandes especialistas y observadores del quehacer diario de sus hijos.

Reforzar los PRITE, ya que son un factor importante en el desarrollo integral de los niños con discapacidad y auguran una mejor calidad de vida de los mismos.

\section{REFERENCIAS}

Palacios Rizzo, A. y Bariffi, F. (2007). La discapacidad como una cuestión de derechos humanos Una aproximación a la Convención Internacional sobre los Derechos de las Personas con Discapacidad. Ediciones Cinca, S. A.

Degener T. \& Quinn, G. (2000). A survey of international, comparative and regional Disability Law Reform. Presentado en: From Principles to Practice, an International Disability law and Policy Symposium, 22 al 25 de octubre, organizado por DREDF. Disponible en: http://www. nuigalway.ie/law /Common\%20Files / Disability\%20Research\% 20Unit/

Declaración Universal de los Derechos Humanos.

García Ruiz, A. y Fernández A. (2005). La inclusión para las personas con discapacidad: entre la igualdad y la diferencia. Revista Ciencia. Salud / 3 (2): 235-246, julio-diciembre. Bogotá.

Casado D, y Egea C. (2000). Las estrategias para el cambio pro inclusión de las personas con discapacidad. España; Recuperado de: http://usuarios. discapnet.es/disweb2000/art/estrategias.htm

Portal web UNICEF América Latina y el Caribe. https:/ /www.unicef.org/ lac/ni\%C3\%B1os-y-ni\%C3\%B1as-con-discapacidad

LEY № 30364, Ley para Prevenir, Sancionar y Erradicar la Violencia Contra las Mujeres y los Integrantes del Grupo Familiar. 
http://www.perueduca.pe/foro/-/message_boards/message/31303036.

Ley 28084, Ley General de Educación.

Decreto Supremo Nro.011-2012-ED, Reglamento de la Ley General de Educación

Ley № 27337.- Aprueba el Nuevo Código de los Niños y Adolescentes NACIONES UNIDAS (s/f). Personas con Discapacidad Departamento de Asuntos Económicos y Sociales. Disponible en: https://www. un.org/development/desa/disabilities-es/discapacidad-desastresnaturales-y-situaciones-de-emergencia.html

Convención de las Naciones Unidas Sobre los Derechos del Niño.

Convención de las Naciones Unidas Sobre los Derechos de las Personas con Discapacidad.

Instituto Nacional de Defensa Civil - INDECI (2016). Derechos de la Persona con Discapacidad en situaciones de emergencia y desastres. Lima: Dirección de Desarrollo y Fortalecimiento de Capacidades Humanas.

Fecha de recepción: 20 de octubre de 2019

Fecha de aceptación: 04 de noviembre de 2019 